\title{
Performance of Beamforming for Smart antenna using Traditional LMS algorithm for various parameters
}

\author{
${ }^{1}$ Vishal V Sawant, ${ }^{2}$ Mahesh Chavan \\ KITCOE ,Kolhapur, Maharashtra, India, \\ maheshpiyu@gmail.com
}

Received: February 20, 2021. Revised: April 5, 2021. Accepted: April 8, 2021. Published: April 14, 2021.

\begin{abstract}
Adaptive signal processing sensor arrays, known also as smart antennas. The smart antenna adaptive algorithms achieve the best weight vector for beam forming by iterative means. The Least Mean Square (LMS) algorithm, is an adaptive algorithm .LMS incorporates an iterative procedure that makes successive corrections to the weight vector in the direction of the negative of the gradient vector which eventually leads to the minimum mean square error. Beam forming is directly determined by the two factors. The performance of the traditional LMS algorithm for different parameters is analysed in this paper. This algorithm can be applied to beam forming with the software Matlab. The result obtain can achieve faster convergence and lower steady state error. The algorithms can be simulated in MATLAB 7.10 version.
\end{abstract}

Keywords- Smart antenna, LMS algorithm, Direction of arrival(DOA)

\section{INTRODUCTON}

Throughout the world, there is significant research and development on smart antennas for wireless systems. This is because smart antennas have tremendous potential to enhance the performance of future generation wireless systems as evidenced by the antennas' recent deployment in many systems. There are two basic types of smart antennas. The first type is the phased array or multibeam antenna, which consists of either a number of fixed beams with one beam turned on towards the desired signal or a single beam (formed by phase adjustment only) that is steered toward the desired signal. The other type is the adaptive antenna array is an array of multiple antenna elements, with the received signals weighted and combined to maximize the desired signal to interference plus noise power ratio. This essentially puts a main beam in the direction of the desired signal and nulls in the direction of the interference. A smart antenna is therefore a phased or adaptive array that adjusts to the environment. That is, for the adaptive array, the beam pattern changes as the desired user and the interference move; and for the phased array the beam is steered or different beams are selected as the desired user moves. Nearly every company the WTEC panel visited is doing significant work in smartantennas. Indeed, some companies placed strong emphasis on this research. In particular, researchers at NEC and NTT stated that they felt that smart antenna technology was the most important technology for fourth generation cellular systems. Researchers at Filtronics and other companies agreed that smart antenna technology was one of the key technologies for fourth generation systems.

A smart antenna system at the base station of a cellular mobile system is depicted in Fig 1. It consists of a uniform linear antenna array for which the current amplitudes are adjusted by a set of complex weights using an adaptive beamforming algorithm. The adaptive beamforming algorithm optimizes the array output beam pattern such that maximum radiated power is produced in the directions of desired mobile users and deep nulls are generated in the directions of undesired signals representing co-channel interference from mobile users in adjacent cells.

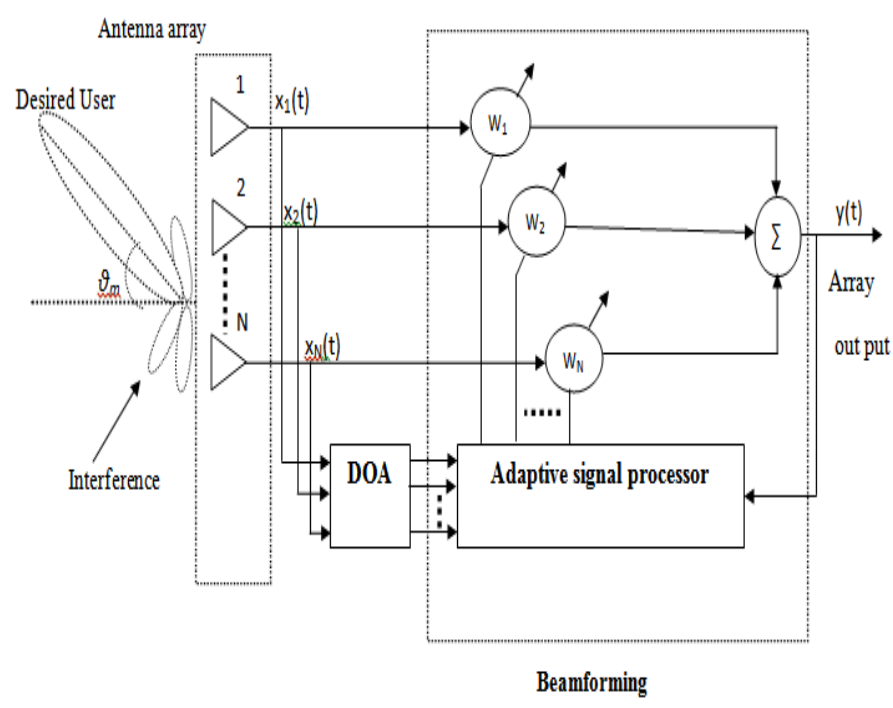

Fig1Block diagram of smart antenna

Prior to adaptive beamforming, the directions of users and interferes must be obtained using a direction-of- arrival (DOA) estimation algorithm.

The goal of direction-of-arrival (DOA) estimation is to use the data received on the downlink at the base-station sensor array to estimate the directions of the signals from the desired mobile users as well as the directions of interference signals. The results of DOA estimation are then used by to adjust the weights of the adaptive beamformer so that the radiated power is maximized towards the desired users, and radiation nulls are placed in the directions of interference signals. $\mathrm{H}$ ence, a 
successful design of an adaptive array depends highly on the choice of the DOA estimation algorithm which should be highly accurate and robust.

Adaptive smart antennas are the array antennas whose radiation pattern is shaped according to some adaptive algorithms. Smart essentially means computer control of the antenna performance. The smart antenna radiation pattern directs the beam towards the users of interest only \& nulls toward interference to improve the capacity of cellular system. The adaptive beam forming algorithms takes the fixed beam forming process one step further $\&$ allows for the calculation of continuously updated array weights.

According to signal space information smart antenna can form directional beam in space with the adaptive beam forming algorithm, achieving that the main beam aims at the direction of the expected signal while the side lobe and nulls aims at the interference. Now many adaptive algorithms have been proposed on smart antenna. The RLS algorithm and LMS algorithm are most commonly used as a adaptive beam forming algorithm.

\section{LMS ALGORITHM}

Consider a uniform linear Array (ULA) with $\mathrm{N}$ isotropic elements, which forms the integral part of the adaptive beamforming system as shown in the figure below. The output of the antenna array $x(t)$ is given by

$\boldsymbol{x}(\boldsymbol{t})=\boldsymbol{s}(\boldsymbol{t}) \boldsymbol{a}\left(\theta_{0}\right)+\sum_{i=1}^{w_{u}} u_{i}(t) a\left(\theta_{i}\right)+\boldsymbol{n}(\boldsymbol{t})$

As shown below the outputs of the individual sensors are linearly combined after being scaled using corresponding weights such that the antenna array pattern is optimized to have maximum possible gain in the direction of the desired signal and nulls in the direction of the interferers.

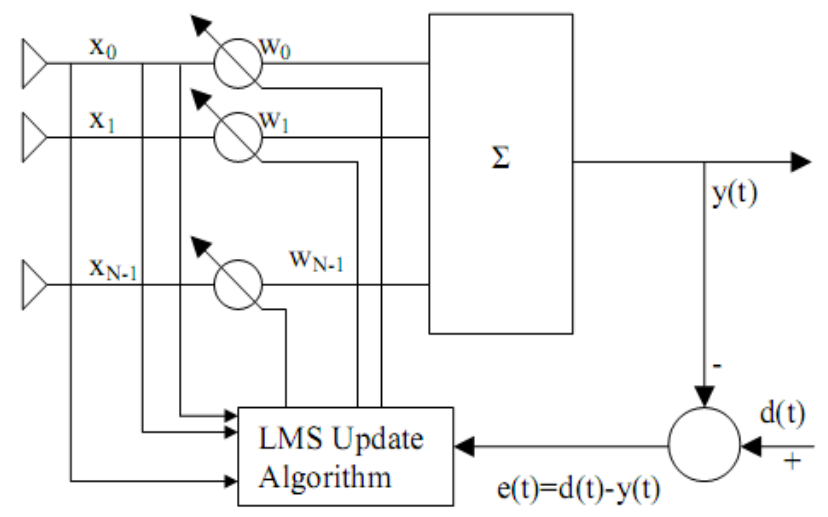

Fig2. LMS adaptive beamforming network

The weights here will be computed using LMS algorithm based on Minimum Squared Error (MSE) criterion. Therefore the spatial filtering problem involves estimation of signal $s(t)$ from the received signal $x(t)$ (i.e. the array output) by minimizing the error between

the reference signal $d(t)$ which closely matches or has some extent of correlation with the desired signal estimate and the beamformer output $\mathrm{y}(\mathrm{t})$. This is a cl assical Weiner filtering problem for which the solution can be iteratively found using the LMS algorithm.

LMS algorithm formulation- (All signals are represented by their sample values) from the method of steepest descent, the weight vector equation is given by

$w(n+1)=w(n)+\frac{1}{2} \mu\left[-\nabla\left(E\left\{e^{2}(n)\right\}\right)\right]$

Where $\mu \mathrm{i}$ s the step-size parameter and controls the convergence chachteristics of the LMS algorithm; $\mathrm{e}^{2}(\mathrm{n})$ is the mean square error between the beamformer output $y(n)$ and the reference signal which is given by,

$e^{2}(n)=\left[d^{*}(n)-w^{h} x(n)\right]^{2}$

The gradient vector in the above weight update equation can be computed as

\section{$\mathbb{V}_{W}\left(E\left\{e^{2}(n)\right\}\right)=-2 r+2 R w(n)$}

In the method of steepest descent the biggest problem is the computation involved in finding the values $r$ and $R$ matrices in real time. The LMS algorithm on the other hand simplifies this by using the instantaneous values of covariance matrices $r$ and $R$ instead of their actual values i.e.

$\mathrm{s}(\mathrm{t})$ denotes the desired signal arriving at angle $\theta_{\mathrm{o}}$ and $\mathrm{u}_{\mathrm{i}}(\mathrm{t})$ denotes interfering signals arriving at angle of incidences $\theta_{\mathrm{i}}$ respectively. $\mathrm{a}\left(\theta_{\mathrm{o}}\right)$ and $\mathrm{a}\left(\theta_{\mathrm{i}}\right)$ represents the steering vectors for the desired signal and interfering signals respectively. Therefore it is required to construct the desired signal from the received signal amid the interfering signal and additional noise $\mathrm{n}(\mathrm{t})$.

$$
\begin{aligned}
& R(n)=x(n) x^{h}(n) \\
& r(n)=d^{*}(n) x(n)
\end{aligned}
$$

Therefore the weight update can be given by the following equation,

$$
\begin{aligned}
& w(n+1)=w(n)+\mu x(n)\left[d^{*}(n)-x^{n}(n) w(n)\right] \\
& =w(n)+\mu x(n) e^{*}(n)
\end{aligned}
$$

The LMS algorithm is initiated with an arbitrary value w(0) for the weight vector at $n=0$. The successive corrections of the weight vector eventually leads to the minimum value of the mean squared error. 
Therefore the LMS algorithm can be summarized in following equations;

output,

$$
y(n)=w^{n} x(n)
$$

Error,

$$
e(n)=d^{*}(n)-y(n)
$$

Weight, $\quad w(n+1)=w(n)+\mu x(n) e^{*}(n)$

\section{CONVERGENCE AND STABILITY OF THE LMS ALGORITHM}

The LMS algorithm initiated with some arbitrary value for the weight vector is seen to converge and stay stable for

$$
0<\mu<\frac{1}{\lambda_{\max }}
$$

Where $\lambda \max$ is the largest eigenvalue of the correlation matrix $\mathrm{R}$. The convergence of the algorithm is inversely proportional to the eigenvalue spread of the correlation matrix R.When the eigenvalues of $R$ are widespread, convergence may be slow. The eigenvalue spread of the correlation matrix is estimated by computing the ratio of the largest eigenvalue to the smallest eigen value of the matrix. If $\mu$ is chosen to be very small then the algorithm converges very slowly. A large value of $\mu$ may lead to a faster convergence but may be less stable around the minimum value. One of the literatures [will provide reference number here] also provides an upper bound for $\mu$ based on several approximations as $\mu=1 /(3 \operatorname{trace}(\mathrm{R}))$.

\section{SIMULATION RESULTS}

\section{Simulation results of Direction of arrival for} traditional LMS is shown.

A GUI has been built to ease the simulation. A Layout of the GUI is depicted in Figure 3. The user can input the signal parameters including angle(s) of arrival, their signal power, number of data snapshots, element of linear array a nd distance between elements.

Case 1: Figure 3, It is considered that there are three desired users with signals arriving at angles -40,0,60 degrees with the different signal strength .Number of snapshots are 2000, number of array elements $\mathrm{N}=16$ with spacing between elements, $\mathrm{d}=0.5 \lambda$

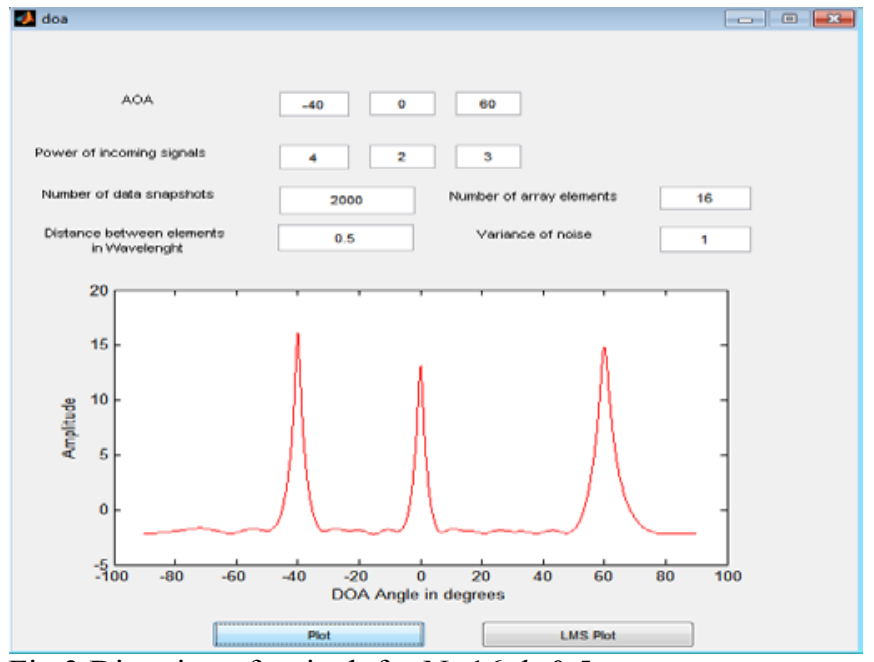

Fig 3 Direction of arrival for $\mathrm{N}=16, \mathrm{~d}=0.5$

Case 2: Figure 4, It is considered that there are three desired users with signals arriving at angles -40,0,60 degrees with the different signal strength .Number of snapshots are 2000, number of array elements $\mathrm{N}=16$ with spacing between elements, $\mathrm{d}=0.2 \lambda$

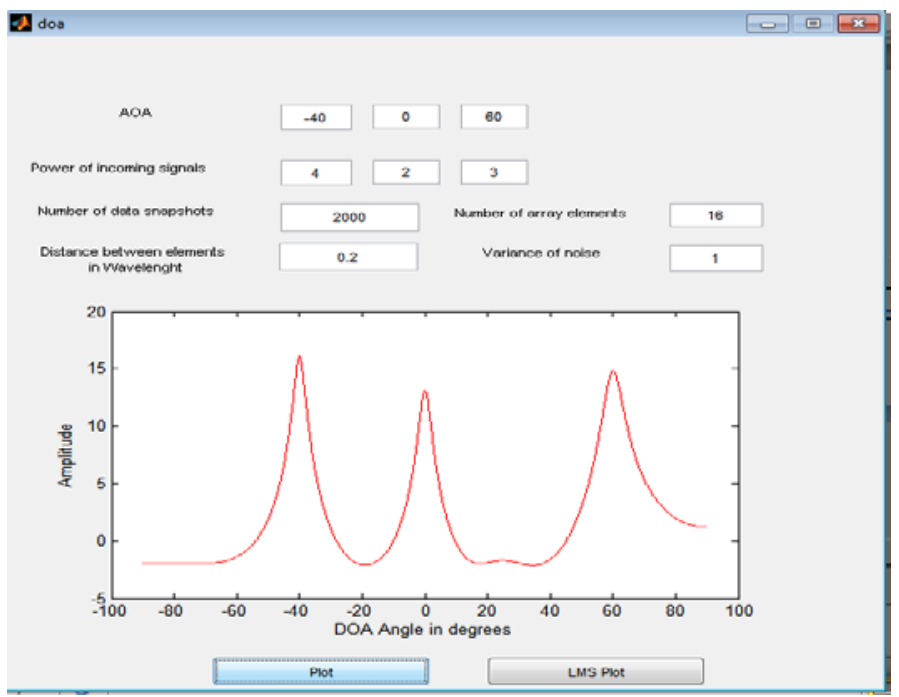

Fig 4Direction of arrival for $\mathrm{N}=16, \mathrm{~d}=0.2$

Case 3: Figure 5, It is considered that there are three desired the different signal strength .Number of snapshots are 2000, number of array elements $\mathrm{N}=8$ with spacing between elements, $\mathrm{d}=0.2 \lambda$ 


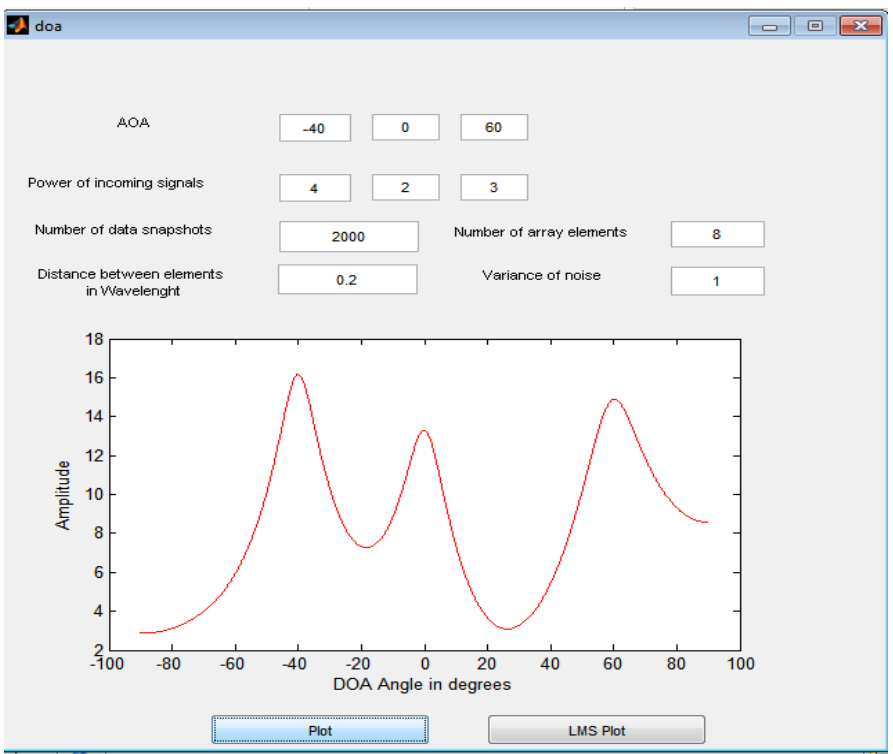

Fig 5Direction of arrival for $\mathrm{N}=8, \mathrm{~d}=0.2$

It has been noticed from results that sharper beams are $\mathrm{d}$ irected towards desired signals as more elements are used in antenna array.Also, spacing between array elements has an effect on beamformer performance such that very small or very large spacing between array elements can degrade beamformer performance . From different numerical calculation it has been observed that element spacing of $0.5 \lambda$ is a good value

\section{Simulation results of Error curve for traditional LMS is shown.}

Figure 6 shows the error curve for desired users with signals arriving at angles $-40,0,60$ degrees with the different signal strength .Number of snapshots are 2000, number of array elements $\mathrm{N}=16$ with spacing between elements, $\mathrm{d}=0.5 \lambda$

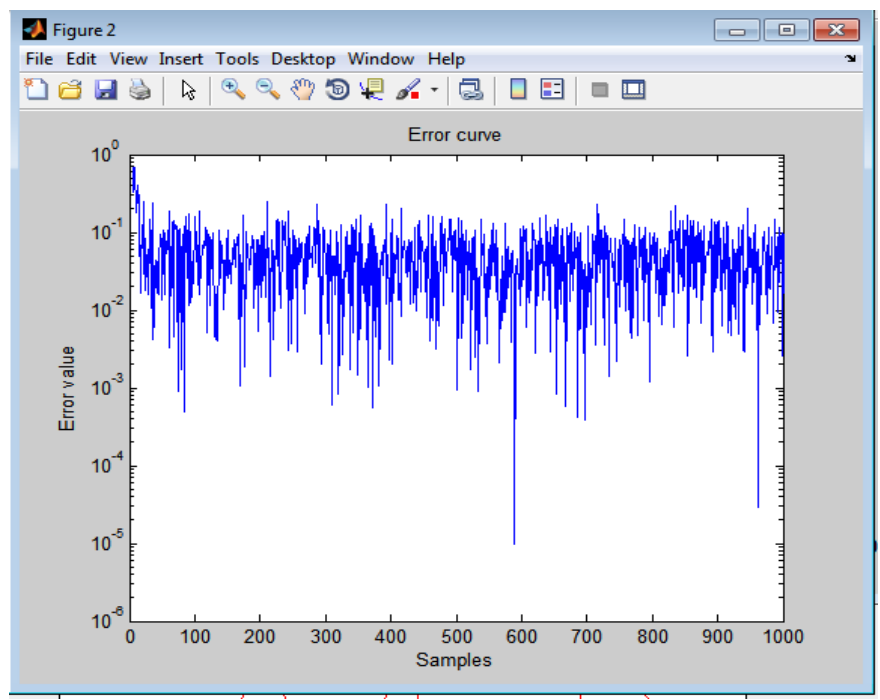

Fig6 Error curve $\mathrm{N}=16, \mathrm{~d}=0.5 \lambda$
Figure 7 shows the error curve for desired users with signals arriving at angles --40,0,60 degrees with the different signal strength . Number of snapshots are 2000, number of array elements $\mathrm{N}=16$ with spacing between elements, $\mathrm{d}=0.2 \lambda$

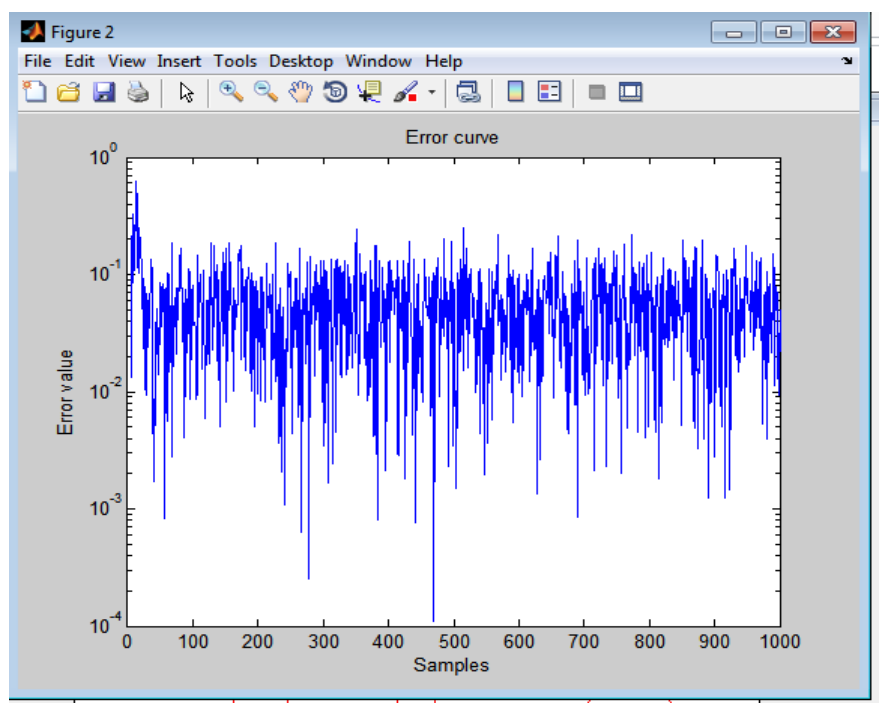

Fig 7 Error curve $\mathrm{N}=16, \mathrm{~d}=0.2 \lambda$

Figure 8 shows the error curve for desired users with signals arriving at angles $-40,0,60$ degrees with the different signal strength .Number of snapshots are 2000, number of array elements $\mathrm{N}=8$ with spacing between elements, $\mathrm{d}=0.2 \lambda$

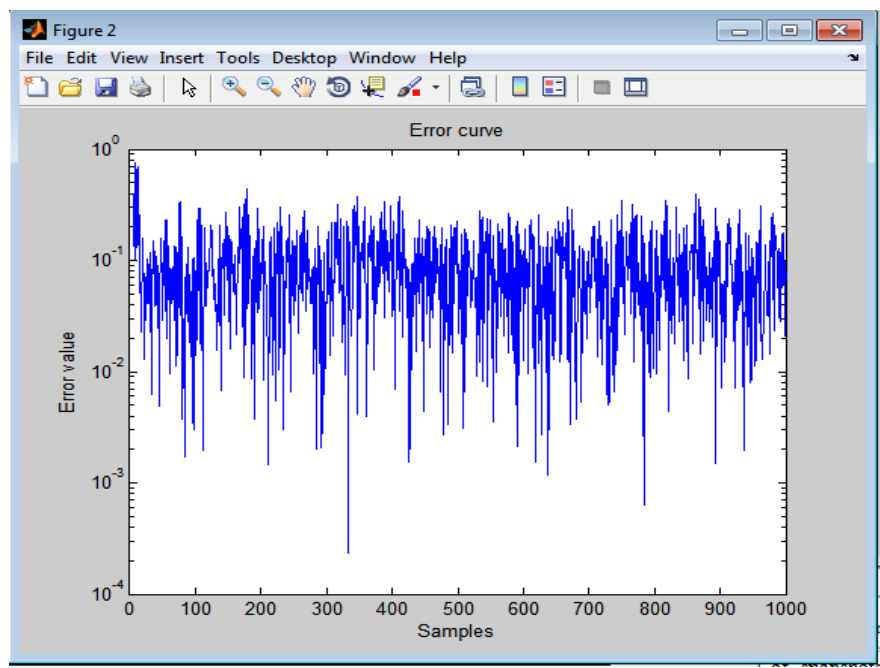

Fig 8 Error curve $\mathrm{N}=08, \mathrm{~d}=0.2 \lambda$

It has been noticed from results that the error curve plot for case 1 shows system error is less and steady for desired users with signals arriving at angles $-40,0,60$ de grees with the different signal strength . Number of snapshots are 2000, number of array elements $\mathrm{N}=16$ with spacing between elements, $\mathrm{d}=0.5 \lambda$.

Simulation results of radiation pattern for traditional LMS is shown 
Figure 9 shows the radiation pattern for desired users with signals arriving at angles $-40,0,60$ degrees with the different signal strength .Number of snapshots are 2000, number of array elements $\mathrm{N}=16$ with spacing between el ements, $\mathrm{d}$ $=0.5 \lambda$

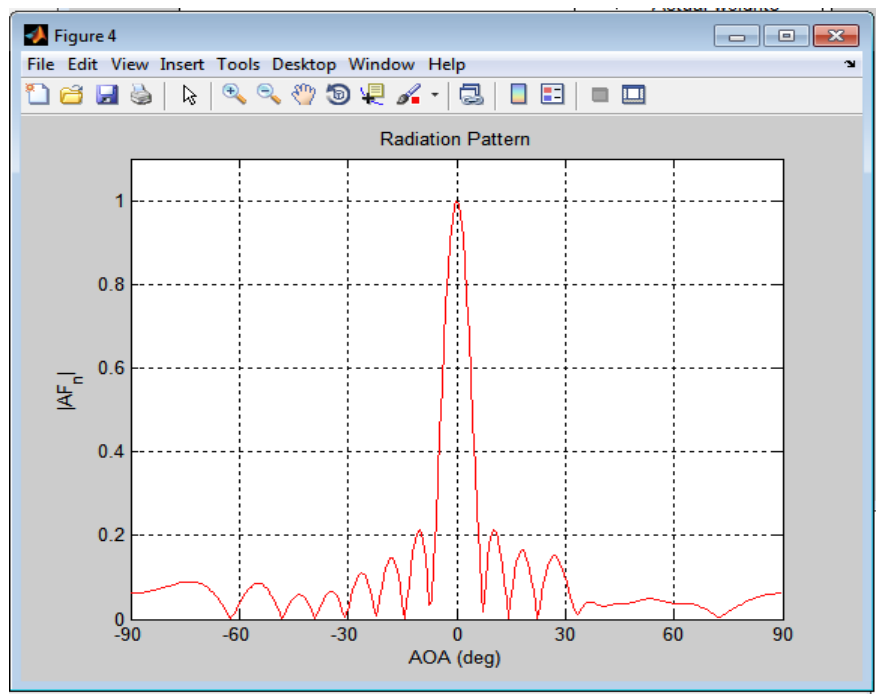

Fig 9 Radaitionpattern for $\mathrm{N}=16, \mathrm{~d}=0.5 \lambda$

Figure 10 shows the radiation pattern for desired users with signals arriving at angles --40,0,60 de grees with the different signal strength . Number of snapshots are 2000, number of array elements $\mathrm{N}=16$ with spacing between elements, $\mathrm{d}=0.2 \lambda$

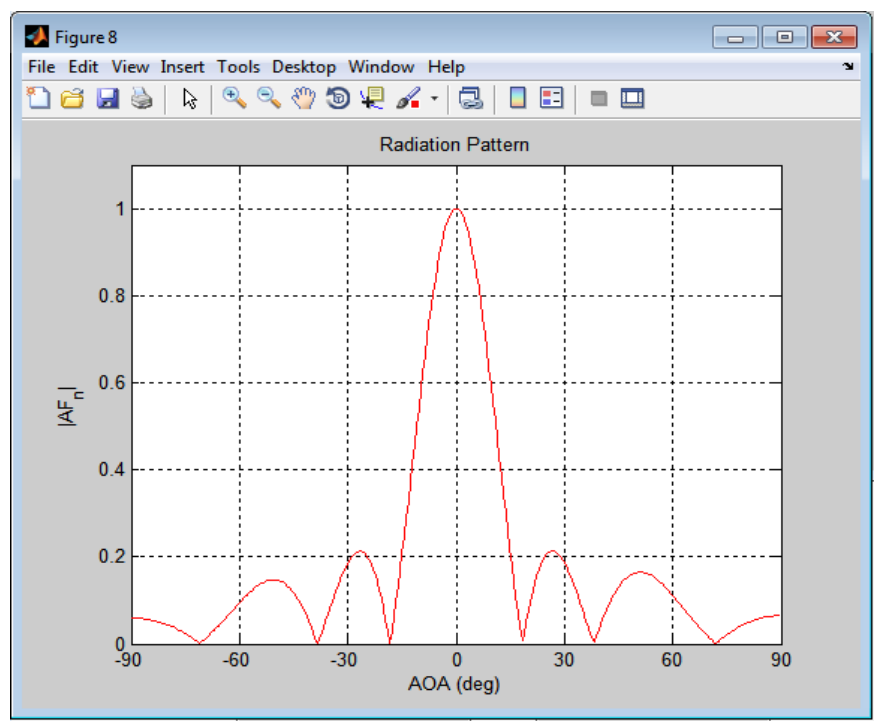

Fig 10 Radaition pattern for $\mathrm{N}=16, \mathrm{~d}=0.2 \lambda$

Figure 11 showsradiation pattern for desired users with signals arriving at angles $-40,0,60$ degrees with the different signal strength .Number of snapshots are 2000, number of array elements $\mathrm{N}=8$ with spacing between el ements , $\mathrm{d}$ $=0.2 \lambda$

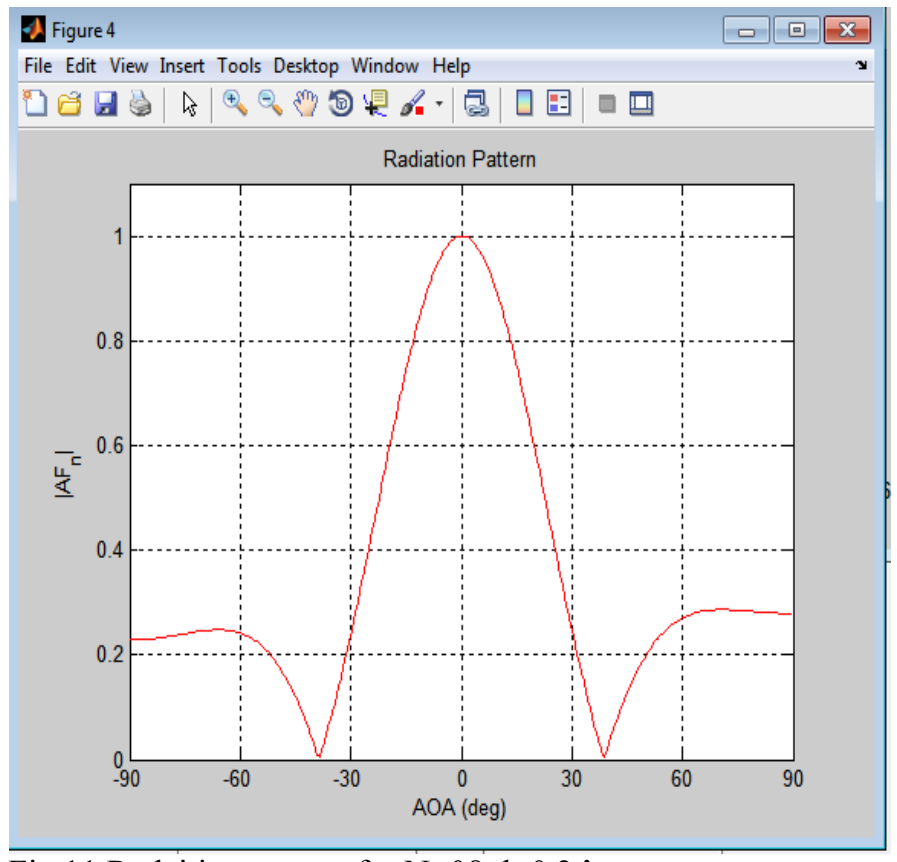

Fig 11 Radaition pattern for $\mathrm{N}=08, \mathrm{~d}=0.2 \lambda$

It has been noticed from results that the radiation patternfor case 1showshas faster convergence rate and can form deeper nulls in the direction of interference.for desired users with signals arriving at angles $-40,0,60$ degrees with the different signal strength . Number of snapshots are 2000, number of array elements $\mathrm{N}=16$ with spacing between elements , $\mathrm{d}$ $=0.5 \lambda$.

\section{CONCLUSIONS}

This paper discussed the performance ofadaptivebeamforming traditional LMS algorithm used in smart antenna. For different number of array element and different spacing between elements are considered for simulation. This paper studied the results for direction of arrival for traditional LMS it was found that for case 1 sharper beams are directed towards desired signals as more elements are used in antenna array, the error curve plot for case 1 shows system error is less and steady, the radiation pattern for case 1 showshas faster convergence rate and can form deeper nulls in the direction of interference. The result obtain achieves faster convergence and lower steady state error

\section{REFERENCES}

[1] S hiann-Jeng $\mathrm{Yu}$ and Ju-Hong Lee, “Adaptive Array Beamforming Based on an Efficient Technique”, IEEE trans. Antennas and Propagation. 1996,44(8) 10941101.

[2] L. S. Reed, J. D. Mallett, "Rapid Convergence Rate in Adaptive Arrays", IEEE trans. A coustics Aerospace and Electronic Systems. 1974, 10(6):853-863. 
[3] K omal R. Borisagar and Dr.G.R.Kulkarni, "Simulation and Comparative Analysis of LMS and RLS Algorithms Using Real Time Speech Input Signal",.Global Journal of Researches in Engineering Page 44 Vol.10 Issue 5 (Ver1.0)October2010.

[4] R aymond G Kwang and Edward W.Johnston, "Variable Step Size LMS algorithm",IEEEtrans.Signal Processing vol 40 No 7, July 1992.

[5] SidiBahri an dFethi Bendimerad, "Performance of Adaptive Beamforming Algorithm $f$ or LMSMCCDMA MIMO Smart Antennas", The International Arab Journal of Information Technology, Vol. 6, No. 3, July 2009.

[6] Seungwon Choi and Donghee Shim, "A Novel Adaptive Beamforming Algorithm for a Smart Antenna System in a CDMA Mobile Communication Environment",IEEE trans vehicular technology, Vol. 49, No. 5, September 2000.

[7] L i Cun-Wu, Lin Chun-Sheng. Discussion on Variable Step LMS Algorithms [J]Ship and Electronic Engineering. 2008.05

\section{Creative Commons Attribution License 4.0} (Attribution 4.0 International, CC BY 4.0)

This article is published under the terms of the Creative Commons Attribution License 4.0

https://creativecommons.org/licenses/by/4.0/deed.en_US 